\title{
Espondilodiscite: Um Diagnóstico Diferencial Raro de Dor Lombar
}

\section{Spondylodiscitis: A Rare Differential Diagnosis of Lumbar Pain}

Ângela Dias Machado ${ }^{1}$, Lara Ribeiro², Ricardo Cardoso ${ }^{3}$, Rosana Silva ${ }^{1}$, Ana Raquel Estebainha ${ }^{1}$

\section{RESUMO}

Espondilodiscite é um processo inflamatório, geralmente infecioso, que acomete os discos intervertebrais e as vértebras associadas. $\bigcirc$ diagnóstico pode ser bastante difícil, devido à raridade da doença, à sintomatologia insidiosa e inespecífica e à alta prevalência de dor lombar na população geral. Descreve-se o caso clínico de um doente do sexo masculino, 59 anos, com dor abdominal nos quadrantes superiores e também desconforto lombar, acompanhada de mal-estar geral. O seu diagnóstico foi atrasado devido à ausência de achados na tomografia computorizada inicial. Trata-se de um caso que pretende alertar os profissionais de saúde para esta entidade. O diagnóstico é baseado sobretudo num elevado índice de suspeição. O tratamento conservador foi suficiente para a completa recuperação do paciente.

PALAVRAS-CHAVE: Discite; Dor Abdominal; Vértebras Lombares

\footnotetext{
1. Interna de Formação Específica em Medicina Geral e Familiar, Agrupamento de Centros de Saúde Alto Ave, USF Afonso Henriques, Guimarães, Portugal. 2. Especialista em Medicina Geral e Familiar, Agrupamento de Centros de Saúde Alto Ave, USF Afonso Henriques, Guimarães, Portugal. 3. Assistente Graduado em Medicina Geral e Familiar, Agrupamento de Centros de Saúde Alto Ave, USF Afonso Henriques, Guimarães, Portugal.

${ }^{\circ}$ Autor (es) (ou seu (s) empregador (es)) Gazeta Médica 2020. Reutilização permitida de acordo com CC BY-NC. Nenhuma reutilização comercial. ${ }^{\bullet}$ Author(s) (or their employer(s)) Gazeta Médica 2020. Re-use permitted under CC BY-NC. No commercial re-use.
} 


\section{ABSTRACT}

Spondylodiscitis is an inflammatory process, usually infectious, that accommodates intervertebral discs and associated vertebrae. The diagnosis can be quite difficult, due to the rarity of the disease, the insidious and nonspecific symptoms, and the high prevalence of low back pain in the general population. We present a case of a 59-year-old male with abdominal and low back pain, accompanied by poor general condition. Its diagnosis was delayed due to the lack of findings on the initial computed tomography. It is a case that alerts health professionals to this entity. The diagnosis is based mainly on a high index of suspicion. Conservative treatment was sufficient for a complete recovery of the patient.

KEYWORDS: Abdominal Pain; Discitis; Lumbar Vertebrae

\section{INTRODUÇÃO}

O termo espondilodiscite engloba osteomielite vertebral, espondilite e discite, uma vez que atinge o disco intervertebral e os corpos vertebrais contíguos. ${ }^{1,2}$ Osteomielite vertebral e discite podem ocorrer simultaneamente (espondilodiscite) ou independentemente. ${ }^{3}$

Trata-se de uma doença rara, mas a sua incidência tem vindo a aumentar devido ao envelhecimento da população, crescente número de pacientes sob medicamentos imunossupressivos ou sob terapia de substituição renal, uso de drogas endovenosas, crescentes taxas de bacteriemia associadas a dispositivos intravasculares ou outras formas de instrumentação, associado, a meios complementares de diagnóstico cada vez mais sensíveis. ${ }^{1,2}$

Apresenta uma incidência de 2,4 casos por cada 100000 habitantes/ano e aumenta com a idade. ${ }^{4}$ Alguns estudos descrevem uma distribuição bimodal da incidência: atinge principalmente pacientes com menos de 20 anos e entre 50-70 anos. ${ }^{1,4}$ Afeta principalmente os indivíduos do sexo masculino com uma proporção de 1,5-2:1. ${ }^{4} \mathrm{~A}$ mortalidade atribuível varia entre os estudos $(2 \%-11 \%){ }^{2}$

Em termos de etiopatogénese, a espondilodiscite pode ter origem piogénica, cujo principal agente etiológico é os Staphylococcus aureus, e granulomatosa, causada por Mycobacterium tuberculosis e/ou Brucella mellitensis, ambas endémicas em Portugal. ${ }^{1}$ As vias de disseminação podem ser hematogénica a partir de um foco bacteriémico distante (70\% dos casos); inoculação direta (por exemplo, após procedimentos raquidianos); ou por contiguidade. ${ }^{1,2}$

A espondilodiscite piogénica tende a afetar doentes com fatores predisponentes ou com um foco infecioso extra-espinhal. ${ }^{3}$ Os fatores de risco para espondilodiscite estão descritos na Tabela 1. Praticamente todos os doentes com espondilodiscite padecem simultaneamente de um ou mais fatores de risco ou comorbilidades. ${ }^{1,3}$
O local mais acometido é a coluna lombar (60\%), seguida pela região torácica (30\%) e cervical (10\%). ${ }^{1}$

A sintomatologia da espondilodiscite é insidiosa, com raquialgia de agravamento progressivo, contínua ou ininterrupta, podendo o doente recorrer múltiplas vezes ao serviço de urgência. ${ }^{1,3-5} \mathrm{O}$ sintoma inicial mais frequentemente referido é lombalgia, sendo que a localização da dor correlaciona-se com o nível vertebral da infeção., $\mathrm{A}$ exacerbação da dor durante a noite deve ser considerada um sinal de alarme. ${ }^{4}$ Contudo, estudos referem que cerca de 15\% dos pacientes não se queixam de dor inicialmente. ${ }^{4}$ A raquialgia pode ter irradiação para a região torácica ou abdominal, consoante a localização. ${ }^{1}$

A ocorrência de febre não é comum, existindo apenas em cerca de $15 \%$ a $50 \%$ dos doentes. ${ }^{1,4}, 6$ Outros sinto-

TABELA 1. Fatores de risco associados ao desenvolvimento de espondilodiscite piogénica. ${ }^{1-3}$

\begin{tabular}{|c|}
\hline Diabetes mellitus \\
\hline $\begin{array}{l}\text { Infeção recente (até } 8 \text { semanas)/Bacteriémia associada a: } \\
\text { - Trato urinário } \\
\text { - Pneumonia } \\
\text { - Tecidos moles, pele } \\
\text { - Abcesso dentário } \\
\text { - Relacionada com acesso vascular (cateter venoso central, } \\
\text { cateter venoso periférico, fístula arteriovenosa) } \\
\text { - Artrite séptica } \\
\text { - Endocardite infeciosa }\end{array}$ \\
\hline Utilizadores de drogas endovenosas \\
\hline Idade $>50$ anos \\
\hline Sexo masculino \\
\hline $\begin{array}{l}\text { Imunodeficiência ou sob terapêutica imunossupressiva/ } \\
\text { corticoterapia }\end{array}$ \\
\hline Cirrose hepática \\
\hline Doença renal crónica \\
\hline Artrite reumatoide \\
\hline Neoplasia \\
\hline Desnutrição (albumina sérica < 3,5 mg/dL e linfócitos < 1500) \\
\hline Tabagismo ou alcoolismo crónico \\
\hline Fratura exposta, trauma penetrante \\
\hline $\begin{array}{l}\text { Cirurgia recente à coluna vertebral (discectomia, artrodese) } \\
\text { ou procedimentos invasivos (punção lombar, raquianestesia) }\end{array}$ \\
\hline
\end{tabular}


mas incluem arrepios, perda de peso, fraqueza e mal-estar. 3,6 Pode estar presente um défice neurológico em 10\% a 20\% dos doentes: diminuição da força muscular, alterações sensitivas, radiculopatia, mielopatia, alterações esfincterianas ou paraplegia. ${ }^{1}$

Devido à sintomatologia fruste e inespecífica na fase inicial, o diagnóstico da espondilodiscite leva em média 11 a 59 dias até ser estabelecido, facto que pode levar a sequelas devastadoras. 1,3,4,6

A avaliação inclui uma anamnese cuidada, exame neurológico completo, avaliação laboratorial e imagiológica, e biópsia. ${ }^{1,2}$

\section{CASO CLÍNICO}

Utente do sexo masculino, 59 anos de idade, raça caucasiana, reformado. Elemento de uma família nuclear, com um índice de Graffar correspondente a classe social média, na fase VI do ciclo de vida familiar de Duvall.

Apresenta antecedentes pessoais de fibrilhação auricular e internamento recente no serviço de Medicina Interna por urossépsis (de 24/11 a 6/12/2019). Medicado habitualmente com rivaroxabano $20 \mathrm{mg}$, sotalol 160 mg e diazepam 5 mg (SOS). Sem antecedentes familiares relevantes. Sem hábitos tabágicos ou etílicos. Sem alteração recente da medicação ou história anterior de alergias.

Do internamento prévio, destaca-se crescimento de Escherichia coli multissensível na urocultura e hemocultura. Foi medicado empiricamente com ceftriaxone; contudo, ao quinto dia de internamento por manutenção de febre, iniciou piperacilina/tazobactam que cumpriu durante 4 dias. Por apirexia sustentada, melhoria dos parâmetros inflamatórios e atendendo ao agravamento da função hepática, ao quarto dia de piperacilina/tazobactam foi descalado para amoxicilina-ácido clavulânico.

Como intercorrência, o utente iniciou ao $3^{\circ}$ dia de internamento queixas de lombalgia de características mecânicas, com ligeira dor à palpação da musculatura local. Realizou tomografia computorizada (TC) lombar (04.12.2019): "Em L3-L4 e L4-L5 observam-se procidências circunferenciais dos discos que moldam o saco tecal e que se insinua na base dos buracos de conjugação, em planos infrajacentes à emergência radicular. Em L5-S1 identifica-se procidência disco-osteofitária de base larga que ocupa parcialmente os buracos de conjugação e que contacta as raízes $L 5$, sem sinais seguros da sua compressão. Artroses posteriores em L4-L5 e L5-S1". Teve alta clínica no dia 6 dezembro 2019, com indicação de cumprir mais 4 dias de antibioterapia.
No dia 6 janeiro de 2020, recorreu a consulta aberta na USF por dor abdominal nos quadrantes superiores de intensidade elevada, com 3 dias de evolução, irradiação dorsal, agravada pela dorsiflexão e pelos movimentos. Não tinha associação com os alimentos e persistia durante a noite. Associadamente referia astenia, mialgias, anorexia, náuseas, hipersudorese noturna e perda ponderal considerável desde o internamento por urosépsis (15 kg em 1,5 meses). Descreveu picos febris nos 3 dias prévios à consulta. Relativamente à lombalgia que iniciou no internamento, referia melhoria parcial da dor, estando com intensidade ligeira, sem agravamento com a palpação e a fazer tratamento fisiátrico. Negou outras queixas, nomeadamente, sintomas urinários, respiratórios, vómitos ou diarreia.

Ao exame objetivo, apresentava um aspeto pálido e emagrecido, estava hemodinamicamente estável, apirético e eupneico. Ao exame abdominal de destacar palpação difusamente dolorosa, sobretudo nos quadrantes superiores, sem defesa ou outros sinais de irritação peritoneal e sem alterações à auscultação/percussão. Apresentava ainda dor muito discreta na palpação da coluna dorso-lombar e sinal de Murphy renal duvidoso bilateralmente.

O doente foi referenciado pela sua médica de família para o serviço de urgência (SU) do hospital de referência. O exame objetivo era sobreponível, sendo que não apresentava alterações no exame neurológico sumário. Analiticamente sem leucocitose e proteína C reativa de 33 mg/dL. Realizou TC abdominal que descreve: "Irregularidades das placas basais de D9-D10, com aumento de partes moles e pequenas coleções subjacentes, podendo traduzir uma eventual espondilodiscite, onde eventual estudo complementar por ressonância magnética (RM) poderá fornecer informações adicionais, caso haja indicação clínica. Rins com topografia e morfologia preservados. Quistos corticais aparentemente simples em ambos os rins, o dominante à esquerda com 36 mm." (Fig.s 1 e 2).

O doente foi internado no Serviço de Ortopedia, tendo sido recomendado repouso no leito e tratamento conservador com antibioterapia endovenosa (segundo antibiograma da hemocultura do internamento prévio: levofloxacina e vancomicina). A hemocultura realizada antes da antibioterapia foi negativa e as serologias víricas negativas.

Realizou RM que confirmou: "sinais sugestivos de espondilodiscite em D9-D10, definindo-se a destruição das plataformas vertebrais e incipiente componente inflamatório/infecioso epidural anterior que não com- 


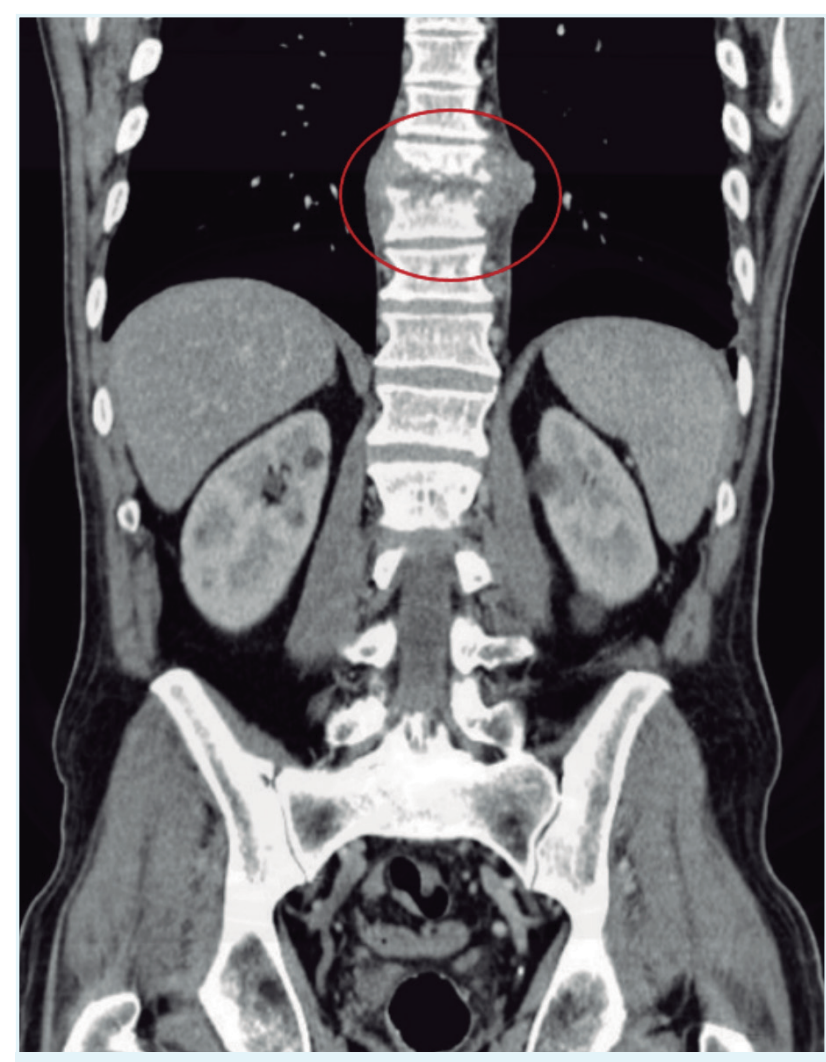

FIGURA 1. Imagem de tomografia computorizada de lesão em disco intervertebral e destruição de corpo vertebral em D9-D10 em plano coronal.

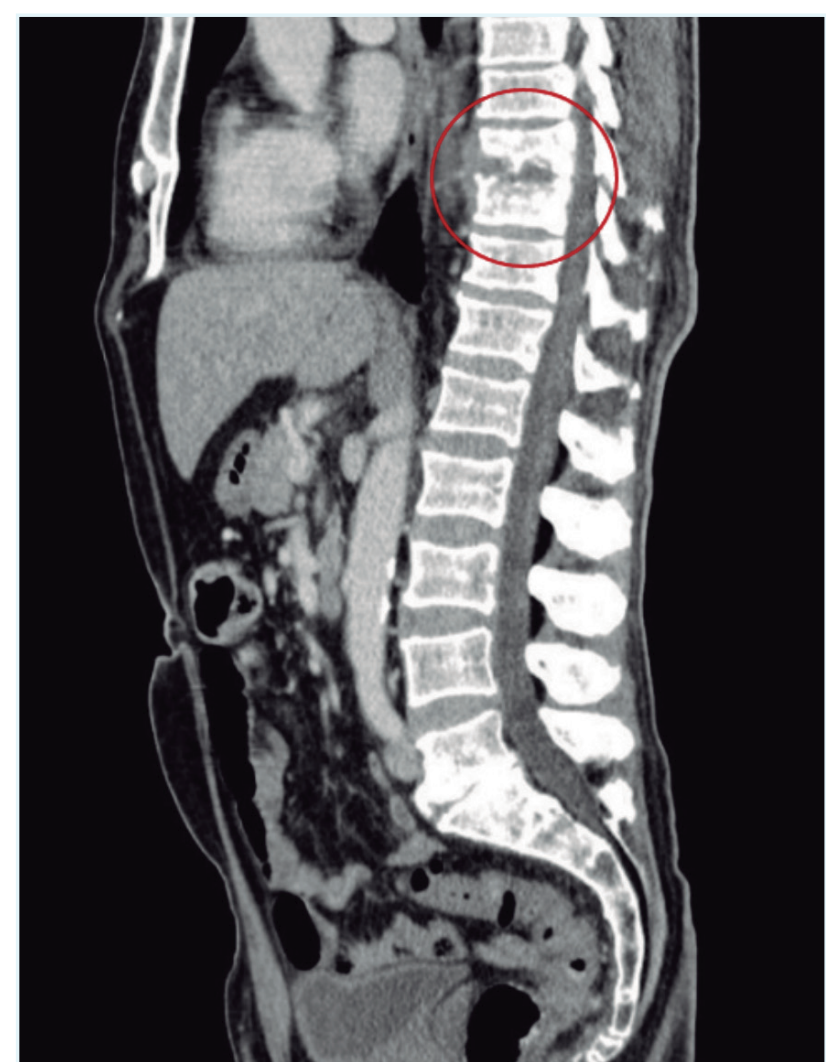

FIGURA 2. Imagem de tomografia computorizada de lesão em disco intervertebral e destruição de corpo vertebral em D9-D10 em plano sagital. prime a medula, mas que se estende discretamente aos buracos de conjugação, reduzindo a amplitude foraminal para a emergência das raízes D9. Definem-se ainda componentes inflamatórios/infeciosos paravertebrais bilaterais, esboçando pequenos abcessos.".

Em seguida, o doente foi submetido a biópsia guiada por imagem que descreve: "inexistência de coleções líquidas que traduzam abcessos paravertebrais. Densificação e espessamento dos tecidos moles que não parecem ter natureza líquida (fleimão? massa tecidos moles?)". Foi colhido fragmento de biópsia, cuja análise bacteriológica descreve: "aparentemente amicrobiano, sem crescimento".

Ao nono dia de internamento, iniciou levante com lombostato conforme tolerância, tendo-se denotado uma miopatia de desuso: membros inferiores com força muscular grau 4 global, verticalizando com apoio e fazendo marcha com supervisão/apoio de terceira pessoa, mas com instabilidade.

Por melhoria clínica e analítica, ao $20^{\circ}$ dia de internamento (D20 de levofloxacina + D18 de vancomicina) teve alta para hospitalização domiciliária, com indicação de programa de reabilitação por enfermagem no domicílio e 30 dias de antibioterapia. Na reavaliação pela sua médica de família após um mês da alta, constatou-se resolução do quadro clínico, tendo o doente recuperado o seu funcionamento prévio, sem sequelas.

\section{DISCUSSÃO}

A espondilodiscite no adulto é pouco frequente, mas é uma realidade, sendo essencial pensar nessa hipótese diagnóstica perante um doente com dorsolombalgia.

Tanto o diagnóstico como o tratamento da espondilodiscite permanecem um desafio, mas se atempados, o seu tratamento tem uma taxa de sucesso de $80 \%{ }^{1}$

O diagnóstico deve ser estabelecido com base nas manifestações clínicas, alterações laboratoriais e imagiológicas. ${ }^{1,4}$ A sua dificuldade ocorre devido aos sintomas inespecíficos, ao quadro insidioso e à baixa incidência dessa patologia na população. Além disso, a lombalgia, que costuma ser a queixa principal do paciente, é extremamente prevalente e torna-se um fator de confundimento.

No caso em questão, a TC lombar sem aparentes lesões agudas realizada logo no início da lombalgia, a melhoria parcial da dor dorso-lombar e o quadro de dor abdominal dificultaram a suspeição clínica. 
A presença de alguns sinais de alarme na abordagem da lombalgia, nomeadamente o caráter recorrente, a evolução superior a quatro semanas, a febre, a exacerbação noturna, a ausência de alívio com o repouso ou decúbito e a perda de peso inesperada, fizeram com que o envio ao SU fosse esclarecedor no diagnóstico. Outros sinais de alarme que se deve ter também em atenção na abordagem deste sintoma são a alteração da postura ou da marcha, a presença de défice neurológico (falta de força, diminuição da sensibilidade) e a disfunção esfincteriana, que obrigam sempre a exclusão de patologia infeciosa e patologia infiltrativa ou tumoral. .,6 $^{1,6}$

É necessário um elevado índice de suspeição, devendo ter-se em atenção os casos de lombalgia prolongada, sem resposta à terapêutica instituída, em pacientes idosos ou com fatores de risco enunciados na Tabela 1.1,6

Em termos de meios complementares de diagnóstico, a TC tem uma sensibilidade de $67 \%$ e especificidade de 50\%, mas permite uma avaliação limitada dos tecidos moles. ${ }^{6} \mathrm{O}$ gold-standard para o diagnóstico é a RM, com uma sensibilidade de $96 \%$ e uma especificidade de 92\%.6,7

O diagnóstico microbiológico é o pilar do tratamento da espondilodiscite, sendo obtido através de hemocultura ou por biópsia guiada por TC. ${ }^{6}$

Quando associada a infeções do trato urinário ou respiratório prévias (como no caso clínico descrito), o agente etiológico mais frequente é a Escherichia coli. ${ }^{1}$

Embora exames como a ressonância magnética não estejam sempre indicados na avaliação da lombalgia, a decisão deve ser tomada individualmente. Manter um alto índice de suspeita levará ao diagnóstico imediato e à solicitação de imagens de acompanhamento apropriadas.

O tratamento da espondilodiscite pode ser conservador ou cirúrgico. $\bigcirc$ tratamento conservador está indicado se infeção recente, inexistência de défice neurológico e comorbilidades que impeçam uma cirurgia.1,3 A intervenção cirúrgica tem indicação quando ocorre défices neurológicos progressivos, deformidade espinhal ou instabilidade com ou sem dor associada apesar da terapêutica antimicrobiana adequada. ${ }^{3,8}$

Não há consenso sobre a duração da antibioterapia, mas existe evidência que 12 ou mais semanas de tratamento estão associadas a menores taxas de recorrência. ${ }^{3} \mathrm{~A}$ antibioterapia dirigida consiste em 4 a 6 semanas de tratamento endovenoso até melhoria dos sintomas; deve posteriormente ser convertida para a via oral e assim mantida por um período mínimo de 6 a 12 semanas, sob controlo analítico dos parâmetros de infeção.
O tratamento conservador leva à resolução em cerca de $80 \%$ dos casos, em associação com repouso no leito e imobilização com ortótese, para conforto do doente ou prevenção da deformidade. ${ }^{3}$ A idade do paciente, as comorbilidades e os valores da Velocidade de Sedimentação durante o primeiro mês de tratamento podem ajudar a predizer o resultado dos pacientes após tratamento conservador. ${ }^{3}$

Um programa de tratamento conservador, incluindo antibióticos intravenosos, controlo da dor e fisioterapia, foi suficiente para o doente melhorar a sua condição médica e recuperar a função. A evolução clínica no caso clínico estudado foi favorável.

Sendo a espondilodiscite uma entidade nosológica grave e dependendo a eficácia terapêutica de um diagnóstico e intervenção precoces, torna-se necessária uma grande suspeição clínica e atenção ao aparecimento de sinais de alarme em doentes que muitas vezes se apresentam com uma clínica insidiosa e inespecífica.

\section{RESPONSABILIDADES ÉTICAS}

CONFLITOS DE INTERESSE: Os autores declaram a inexistência de conflitos de interesse na realização do presente trabalho.

FONTES DE FINANCIAMENTO: Não existiram fontes externas de financiamento para a realização deste artigo.

CONFIDENCIALIDADE DOS DADOS: Os autores declaram ter seguido os protocolos da sua instituição acerca da publicação dos dados de doentes.

CONSENTIMENTO: Consentimento do doente para publicação obtido.

PROVENIÊNCIA E REVISÃO POR PARES: Não comissionado; revisão externa por pares.

\section{ETHICAL DISCLOSURES}

CONFLICTS OF INTEREST: The authors have no conflicts of interest to declare.

FINANCING SUPPORT: This work has not received any contribution, grant or scholarship.

CONFIDENTIALITY OF DATA: The authors declare that they have followed the protocols of their work center on the publication of data from patients.

PATIENT CONSENT: Consent for publication was obtained.

PROVENANCE AND PEER REVIEW: Not commissioned; externally peer reviewed. 


\section{REFERÊNCIAS}

1. Costa J, Andrade N, Arcangelo J, Pedrosa C, Figueiroa P. Espondilodiscite Piogénica em Adultos - Diagnóstico e Tratamento. Rev Port Ortop Traumatol. 2015:23:225-35.

2. Sato K, Yamada K, Yokosuka K, Yoshida T, Goto M, Matsubara T, et al. Pyogenic spondylitis: clinical features, diagnosis and treatment. Kurume Med J. 2019;65:83-9. doi: 10.2739/kurumemedj.MS653001.

3. Aljawadi A, Jahangir N, Jeelani A, Ferguson Z, Niazi N, Arnal F,et al. Manangement of pyogenic spinal infection, review of literature. J Orthop. 2019;16:508-512. doi: 10.1016/j. jor.2019.08.014.4.

4. Almansour H, Pepke W, Akbar M. Pyogenic spondylodiscitis. The quest towards a clinical-radiological classification. Orthopade. 2020;49:482-93. doi: 10.1007/s00132-019-03836-0.

5. Davis W, April M, Mehta S, Long B, Shroyer S. High risk clinical characteristics for pyogenic spinal infection in acute neck or back pain: Prospective cohort study. Am J Emerg Med. 2020; 38:491-6. doi: 10.1016/j.ajem.2019.05.025.

6. Marchionni E, Marconi L, Ruinato D, Zamparini E, Gasbarrin A, Viale P. Spondylodiscitis: is really all well defined? Eur Rev Med Pharmacol Sci. 2019;23(2 Suppl.):201-9.

7. Sobottke R, Seifert H, Fätkenheuer G, Schmidt M, Goßmann A, Eysel P. Current diagnosis, and treatment of spondylodiscitis. Dtsch Arztebl Int. 2008;105:181-7. doi:10.3238/arztebl.2008.0181.

8. Berbari EF, Kanj SS, Kowalski TJ, Darouiche RO, Widmer AF, Schmitt SK, et al. Infectious Diseases Society of America (IDSA) Clinical Practice Guidelines for the Diagnosis and Treatment of Native Vertebral Osteomyelitis in Adults. Clin Infect Dis. 2015;61:e26-46. doi:10.1093/cid/civ482. 